\title{
CROSS PLATFORM BASED SMART GREENHOUSE APPLICATION
}

\author{
B. KAYCI ${ }^{1}$, B. CERİT ${ }^{2}$, E. YİĞİT ${ }^{3 *}$ \\ ${ }^{1}$ Safranbolu/Karabük bariskayci@hotmail.com \\ ${ }^{2}$ Safranbolu/Karabük ceritbahtiyar@gmail.com \\ ${ }^{3}$ Safranbolu/Karabük ygtenes44@gmail.com
}

KEY WORDS: Smart Greenhouse, IoT, Firebase, Flutter, Wireless Communication

\begin{abstract}
:
In this study, an application has been made in the field of agriculture over the internet of things that are frequently needed in our lives with the developing technology. Due to the increasing human population and consumption needs, countries have begun use agricultural areas and the energy used in agricultural areas more effectively. It is known that temperature and humidity values are critical in greenhouses used in agricultural areas. For this reason, the temperature and humidity values were measured from four different points of the greenhouse by wireless communication in the greenhouse and these values were sent to the main station, and then the greenhouse supervisors were given the opportunity to monitor instantly with Flutter. In addition, if the temperature in the greenhouse falls below the value determined by the responsible people, the greenhouse workers are warned with the help of an audible warning.
\end{abstract}

\section{INTRODUCTION}

Greenhouse is preferred because it makes the area where the production will be made suitable in terms of weather conditions in case there is no suitable climatic conditions in the place where agricultural products are to be grown. Greenhouses are preferred in regions with unfavorable climatic conditions in terms of climatic conditions across the world (Söyler, O., et al., 2018). Examples of these countries are the Netherlands and the United Kingdom. Since the seasonal conditions are difficult in countries with a cool climate, such as the Netherlands, it has aimed to reach a suitable environment with greenhouses. It is known that they use fully automatic greenhouses by using technology for this. Although Turkey is located in the temperate climate zone, greenhouse cultivation is carried out in order to protect the products from frost disaster (Kendirli, B., and Çakmak, B. 2010).

It has been observed that technology is used to get more benefit from the product in greenhouse activities. There are vital parameters such as temperature and humidity in the greenhouse. In addition to these parameters, systems such as irrigation or ventilation are now vital for the greenhouse industry. It has been seen that such studies have entered the literature under the name of smart greenhouse. There are smart greenhouse applications using different controllers and programming languages. It transfers the image inside the greenhouse made using Raspberry Pi to the internet environment. It has also been stated that it transfers the parameters read from the sensor such as temperature, humidity, air quality, light in the greenhouse to the website environment (BaysaL, K., et al., 2018).

In this study, four modules were placed in the greenhouse. These four different modules transmit the measured temperature and humidity data to the center called the main station. NRF24L01 is widely used in wireless communication. There are usage examples in different fields from unmanned aerial vehicles projects to mobile robots. Drone was used for security purposes in the studies. Motion-detecting sensors using NRF24L01 send this data to the area where the drone is located. In line with the incoming information, the drone takes action and goes to the point where the motion sensor is active (Yiğit, E., and Bayır, R., 2021). In addition, NRF24L01 is preferred because it works with very low energy. In a study, sensors placed in the greenhouse were communicated using NRF24L01. In this study, low consumption is aimed because the energy is provided by batteries. In the study, it was determined that NRF24L01 chips draw 800nA in sleep state, 5-6mA in TX mode, $7-12 \mathrm{~mA}$ in RX mode (Gündoğdu, K. S., and Tezcan, C. S., 2018).

In this study, there is an ESP8266 controller wirelessly communicating with the modules in the greenhouse at the main station. The data coming to the base station is first interpreted by the ATMEGA328P. The received data is also transferred to the ESP8266. After the transferred data enters the internet environment, it is transferred to the mobile application coded for greenhouse managers. Thus, the greenhouse supervisor can control the temperature and humidity values in the greenhouse via the mobile application wherever he is. There are projects in which IoT technology is used. It is used in many areas from smart home technologies to agriculture. There are mobile application projects used to monitor the light level at school entrances and smart home entrances (Cerit, B. and Bayır, R., 2020). RFID cards used in school entrances are widely used. The information on these cards are instantly transferred to the mobile application. Thus, the attendance system can be followed instantly (Jaishree, M., et al., 2020).

\section{MATERIALS AND METHODS}

\subsection{Module Materials}

ATMEGA328P, which is used as a microcontroller in the study, detects the temperature and humidity level of the environment and transfers these detected data to the main station. DHT22 sensor is used to measure temperature and humidity level. The

Corresponding author 
temperature and humidity level of the measured and detected environment is transmitted to the main station with the NRF24L01 + PA + LNA SMA Antenna $2.4 \mathrm{GHz}$ Wireless Communication Module. The block diagram of these modules are given in Figure 2.1.

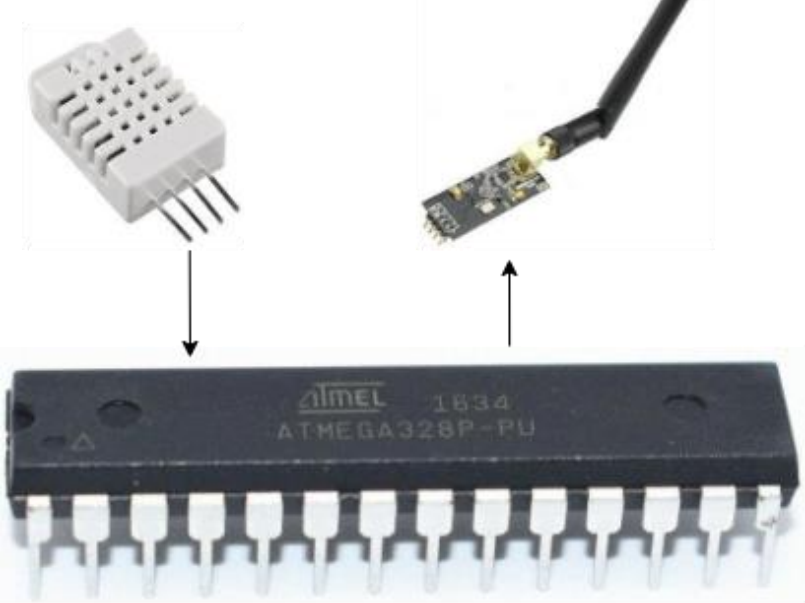

Figure 2.1. Block diagram of these modules.

Actual images of these modules that detect temperature and humidity are given in Figure 2.2.

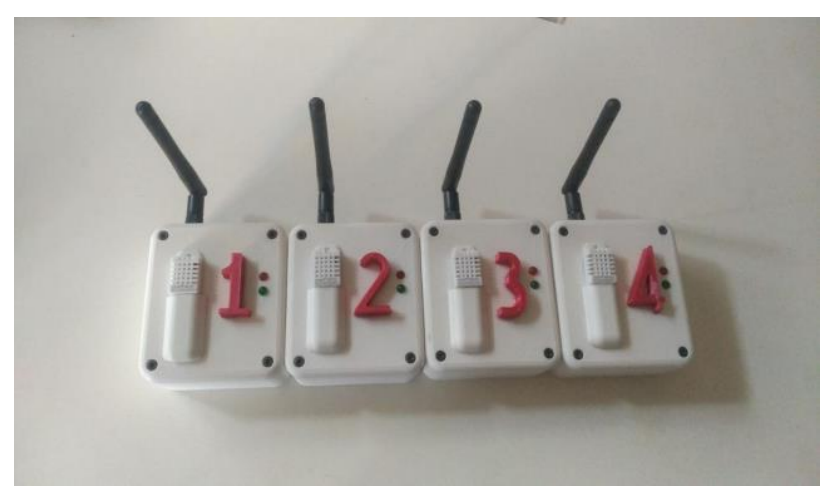

Figure 2.2. Actual view of these modules.

\subsection{Main Station Materials}

Temperature and humidity data from the modules come to the main station. A board has been designed in the main station so that the data can be tracked and stored securely. At the same time, measures were taken against adverse weather conditions with the IP65 certified electrical panel. Electrical Panel is given in figure 2.3.

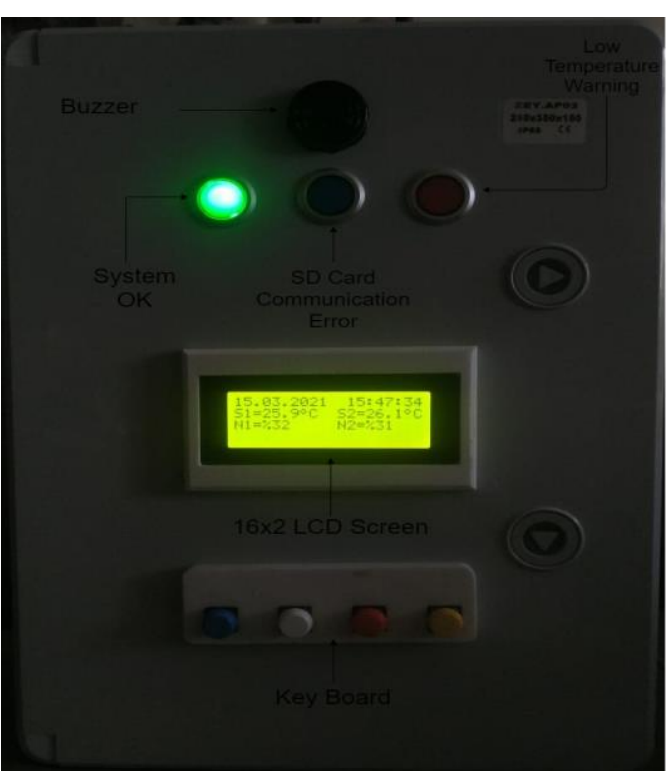

Figure 2.3. The front surface of the electrical panel.

Incoming data traffic can be monitored with the led and screen placed in front of the Electricity Panel. On the front surface of the Electric Panel; Green led indicates that there is no problem in data communication and the temperature is above the specified threshold, blue led indicates that there is a problem in SD card connection, and red led indicates that the measured value falls below the desired temperature (Yıldırım, Ş., et al., 2016).

If the temperature drops below the specified threshold, there is a buzzer to warn the attendants. If it is a $16 \times 2$ LCD screen; By ensuring that the data coming from the modules in the greenhouse can be followed, the threshold value can be increased or decreased at the same time. There is also a keypad to make these operations more convenient. In addition to the keypads with up and down functions, a key is used to confirm and a key to return. Thus, an easy user interface is designed. In addition, there is a main station inside the electrical panel. The block diagram of this base station is given in Figure 2.4.

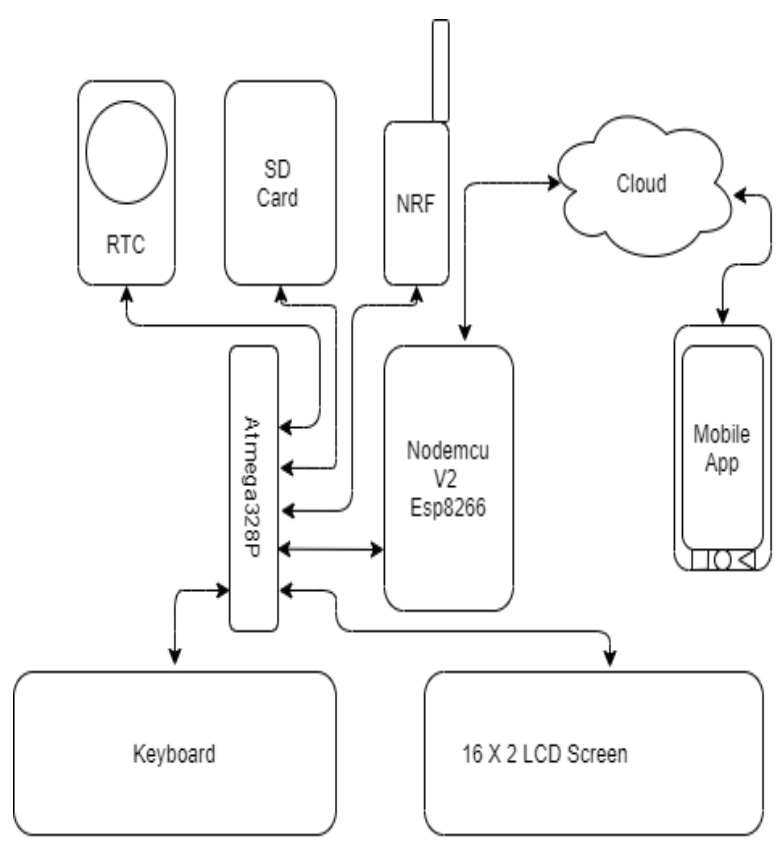

Figure 2.4. Block diagram of the base station. 


\subsection{Method Used in the Module}

In this study, the importance of not falling below a certain degree in the humidity and temperature level of the greenhouse in order to get more efficiency from the products produced in the greenhouse was stated by the experts. The NRF24L01 communicates with the ATMEGA328P in the core of the system. This communication takes place with the SPI protocol. It is known that NRF24L01, which provides high-speed quality communication, can provide a data flow of 2 Mbits per second. There is a wireless data authentication protocol known as SHOCKBURSTTM developed by NORDIC for this product. This protocol is similar to the TCP protocol found in the internet infrastructure. This similarity comes from the fact that the package automatically provides a regular flow of data if necessary. The data obtained from 4 different regions in the inner part of the greenhouse are transmitted to the main station. This transmission takes place in a node structure called nodebase. Node-Base communication structure is given in figure 2.5.

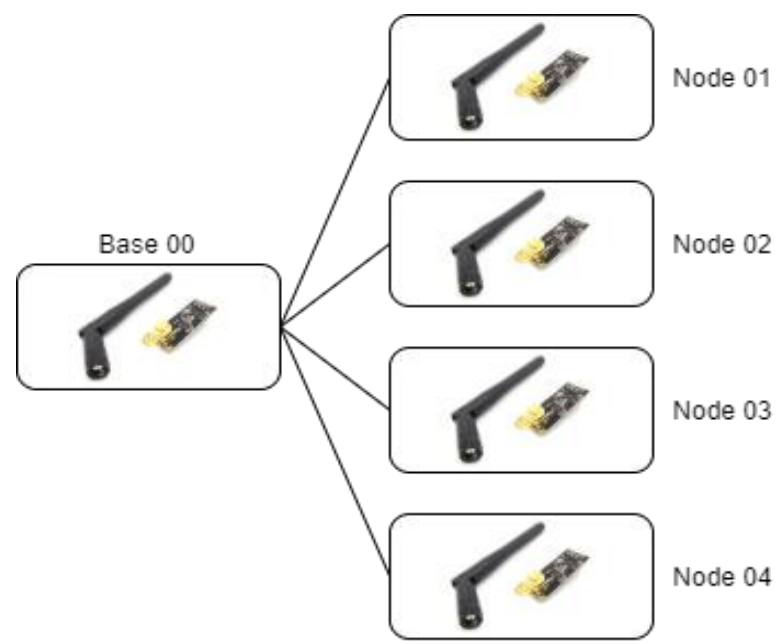

Figure 2.5. Node-Base network.

\subsection{Methods Used in the Base Station}

The method used in the main station is to transfer the data sent to the internet environment to the mobile application. Modules placed in greenhouses transfer the read temperature and humidity values to the main station. These transferred values are transferred to the mobile application via the cloud system so that the administrators can monitor the humidity and temperature in the greenhouse more closely and instantly. This requires a database. The data is first transferred to the database via the internet and then to the mobile application with the necessary code snippets.

In this study, Google Company's Firebase database was used as a database. Firebase Realtime Database is a cloud-supported NoSQL database system. Therefore, it can be controlled with JSON parameters without the need for any SQL query. In addition to data storage, it provides instant data tracking. It stores the data coming to Firebase Realtime Database as a JSON object and creates a tree structure. It becomes a JSON node as data falls into the database. The data read in this flow is interpreted, added, deleted or updated (Kılıç, V., 2021). The Firebase Realtime Database screen of this study is given in Figure 2.6.

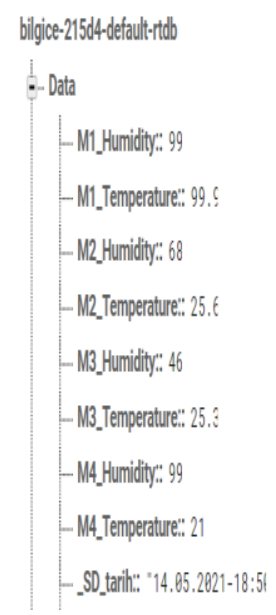

Figure 2.6. Firebase realtime database screen.

Flutter was preferred in this study to write a mobile application. With the mobile application written in Flutter, greenhouse managers or supervisors can view the temperature and humidity in the greenhouse even from kilometers away. In addition, Flutter was launched by Google in 2017. Being cross-platform, Flutter is suitable for writing apps for both Android and IOS. It uses the Dart language on the basis of Flutter, which has a simple interface. The mobile application interface of this study is given in Figure 2.7.

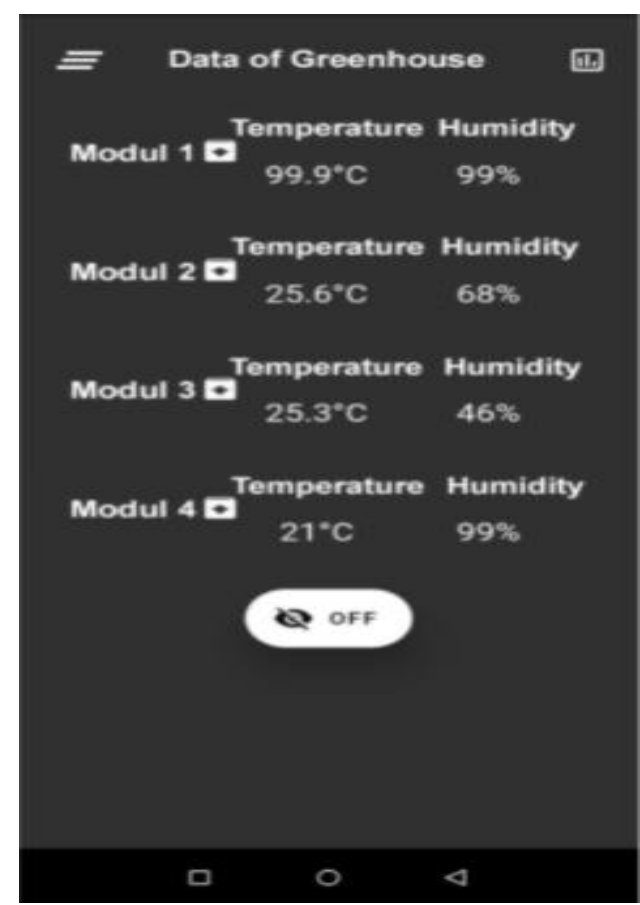

Figure 2.7. Mobile application interface.

\section{EXPERIMENTAL RESULT}

The experiments of this study were carried out in Yazıköy village of Safranbolu District of Karabük Province in Turkey. In the area where there are 8 greenhouses in total, the experiments were carried out for only one greenhouse. 4 modules are placed in the greenhouse, which is $40 \times 8 \mathrm{~m}^{2}$. Thanks to these placed modules, temperature and humidity values were taken from different areas of the greenhouse. Thanks to the $16 \times 2$ LCD Screen available on the panel in the greenhouse area, these 
values obtained were able to follow the greenhouse employees, as well as the greenhouse managers were able to follow up instantly thanks to the mobile application. These values are also recorded at specific time intervals based on date and time. These recorded values were transferred to the Excel table and graphics were obtained. The experiments started in March and continued until mid-May. Due to the favorable weather conditions and energy saving, the operation was put into sleep mode. It is planned to start operating again towards the last months of the autumn season, when the weather conditions are expected to progress negatively in terms of the production of agricultural products. The graphs of four different dates randomly selected from the data records of this study are given in Figure 2.8.
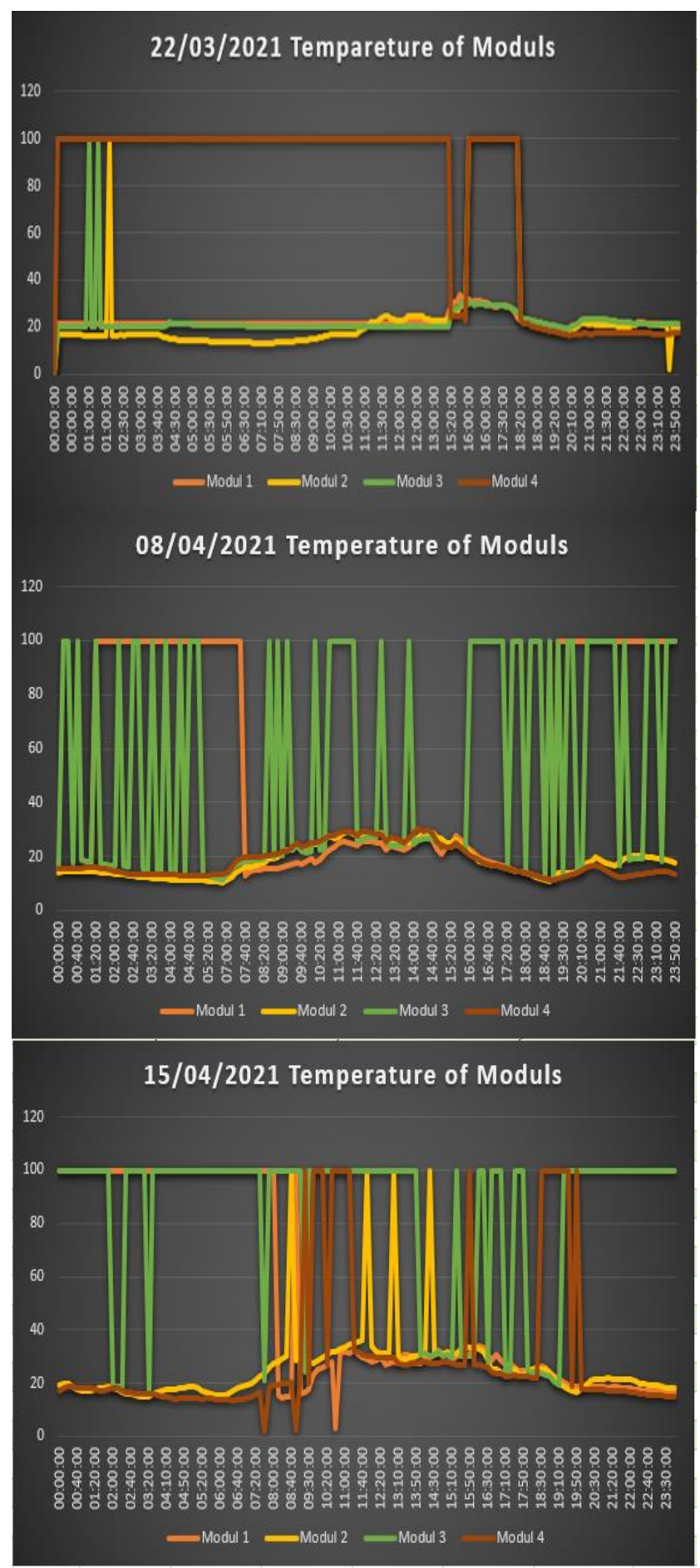

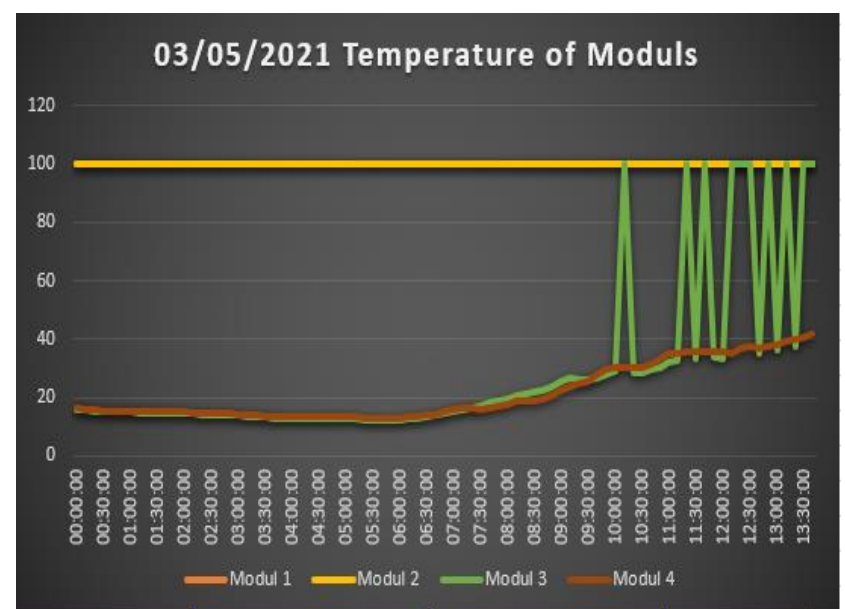

Figure 2.8. Measured values of four modules at four different times.

\section{EVALUATION AND RESULTS}

It has been determined that greenhouses are used in many countries of the world in cold climates or adverse weather conditions. With the use of technology in greenhouses established to meet the needs of consumers, it is aimed to get more performance from the products produced. The main parameters affecting this performance increase are the temperature and humidity data in the greenhouse. The appropriate temperature and humidity level for the product in the greenhouse affects the increase in the number of products. In this study, a Flutter-based smart greenhouse application, which is a cross-platform application, has been made.

It has been seen in the experiments that; Instant monitoring and recording of data in the control of temperature and humidity values provides many inferences for future seasons. In the light of the data obtained, products that are more suitable for the temperature and humidity in the greenhouse can be selected. In addition, a cost calculation opportunity has arisen for the methods used to increase the temperature level. Thus, it was thought that a more planned spending compass could be created.

The materials used in this study are shown in graphs that sometimes miss data. It has been predicted that this problem can be resolved by upgrading the controller used for this. It is thought that the system can continue to operate with much less margin of error if higher quality and price sensors and controllers are preferred.

\section{ACKNOWLEDGEMENT}

This work is supported by Safranbolu Municipality. We would like to thank Elif KÖSE, the mayor of Safranbolu and Mert KOZULU, mayor's advisor for their contributions.

\section{REFERENCES}

Söyler, O., Çakır, M., Kalpakçıŏlu, H., Uğurluay, S., Ömer, E.,Çakır, E., 2018: "Sıcak Havanın Toprak Altına Transferi ve Optimum Ortam Koşullarını Sağlayan Akıllı Sera Otomasyonu Tasarımı". Mesleki Bilimler Dergisi (MBD), 7(1), 1-9. 
Kendirli, B., and Çakmak, B. 2010: "Yenilenebilir Enerji Kaynaklarının Sera Isitmasında Kullanımı”. Ankara Üniversitesi Çevrebilimleri Dergisi, 2(1), 95-103.

Baysal, K., Özcan, M. O., Özdüven, F. F., Beynek, B.2018: "Nesnelerin İnterneti Tabanlı Bir Sera Takip Sistemi". Electronic Journal of Vocational Colleges, 8(2), 49-56.

Yiğit, E., and Bayır, R., 2021: "Wireless Charging System For Multi-Rotor Unmanned Areial Vehicles". V. Uluslararast Battalgazi Bilimsel Çalışmalar Kongresi, Malatya, 137-149

Gündoğdu, K. S., and Tezcan, C. S., 2018: "Sera İçi Kablosuz Sensör Sistemlerinin Pil Performansının Belirlenmesi”, 1st International, 14th National Congress on Agricultural Structures and Irrigation, Antalya, 358-364

Cerit, B., and Bayır, R., (2020): "Deep Learning Based Mask Detection in Smart Home Entries During the Epidemic Process." The International Archives of Photogrammetry, Remote Sensing and Spatial Information Sciences 44, 159-163.

Jaishree, M., Yaminishanmugam, M., Jayarajan, P., Selvy, P. T., 2020: "A Flutter Framework Canary Kit for Miners Using RFID Card", Proteus Journal, 11(12), 44-48

Yıldırım, Ş., Arslan, E., \& Öztürk, O., 2016: "Robot Kolonileri için Arttırılabilir Menzil İletişim Altyapısı", 24th Signal Processing and Communication Application Conference, 117120, DOI:10.1109/SIU.2016.7495690

Kılıç, V., 2021: "Yapay Zeka Tabanlı Akıllı Telefon Uygulaması ile Kan Şekeri Tahmini." Avrupa Bilim ve Teknoloji Dergisi 26: 289-294. 